\title{
TRANSVESTISMO EN PUERTO RICO: TRANSVESTIS DE LA CALLE Y TRANSVESTIS DE TABLA
}

Gloria M. Molina*

\section{Introducción}

$\mathrm{U}$

na de las poblaciones menos estudiadas e investigadas en la realidad social puertorriqueña es la de los transvestis. Esto quizás se deba principalmente a factores culturales y sociales relacionados con el tabú que representa todo aquello relacionado a la sexualidad humana. A lo antes mencionado, también se le suma el hecho de que la conducta del transvestismo es también rechazada y condenada social, política y religiosamente por la sociedad en general. El transvestismo es la conducta de personas que ustilizan vestimentas culturalmente designadas al sexo opuesto ya sea con propósitos sexuales o comerciales. Históricamente se ha identificado al hombre con esta conducta. Se define transvesti como aquel que ejecuta la conducta de vestirse con ropajes del sexo opuesto, además de apropiarse de hábitos sociales del género contrario. Según Noguer (1971), el transvestismo es también denominado "eonismo" en recuerdo a Eón y su historia personal por su afición a vestir ropajes femeninos. Es evidente que la ropa tiene cierto significado como manifestación exterior de la sexualidad. McCary (1996) y Barker (1996), al igual que el DSM III (1992), conceptualizan el

\footnotetext{
* Catedrática, Escuela Graduada de Trabajo Social, Universidad de Puerto Rico.
} 
transvestismo como una desviación sexual que se caracteriza por un deseo compulsivo de usar las prendas del otro sexo. Sin embargo, Hyde (1986), plantea que la conducta del transvestismo es practicada por diversas personas y por diferentes razones. Por ejemplo, existe el que utiliza ropa del sexo opuesto con el objetivo de obtener placer sexual. Por otro lado, Hyde (1986) categoriza como "drag queen" a aquel hombre homosexual que se viste de mujer y percibe como personificador femenino a aquel el hombre que se viste de mujer como parte de un trabajo de entretenimiento o para obtener dinero vendiendo su cuerpo. Algunos visualizan al transvestismo como un arte, esta opinión es compartida por muchos transvestis actores en Puerto Rico que fueron entrevistados por De Los Santos (1995).

Para propósitos del presente estudio, el transvestista se ha clasificado en dos grupos: transvesti de la "calle" y el transvesti de "tabla". El primero se refiere a aquel hombre que utiliza ropaje y adornos propios del sexo contrario con el propósito de prostituirse para satisfacer sus necesidades económicas y/o sexuales. Por otro lado, el transvesti de tabla se refiere al hombre que utiliza el arte de vestirse de mujer para realizar espectáculos y presentaciones artísticas, ya sea con fines lucrativos y/o placer sexual.

En este estudio se exploran las siguientes variables: características sociodemográficas de los transvestis, las relaciones familiares y parentales, características de su clientela o público, prácticas sexuales utilizadas, información recibida sobre SIDA y relaciones de pareja.

Al revisar la literatura sobre investigaciones realizadas en Estados Unidos con esta población, se encontró que no existe una edad fija en la que se comienza a practicar el transvestismo. Bullough y Bullough (1997), encontraron en su estudio con una muestra de 372 transvestis que la mayoría de éstos comenzaron a vestirse de mujer a los ocho (8) años, mientras que un $32 \%$ lo hizo antes de los seis (6). Además, concluyeron que las experiencias sexuales durante la adolescencia y la niñez de estos individuos influyeron en su conducta sexual actual (Bullough y Bullough, 1997). Por otro lado, Peo (1988) encontró en su investigación que los transvestis entrevistados comenzaron a usar prendas femeninas después de los cinco años y no en la pubertad, o más tarde. Algunos transvestis manifestaron que comenzaron desde muy niños y que al utilizar la ropa de su madre o hermana se sentían muy excitados o sexualmente poderosos (Peo, 1988). En la encuesta realizada por Schott (1995) a 85 transvestis se reportó que casi la totalidad de la muestra, o sea, un $97 \%$ expresó haber 
tenido su primera experiencia con vestidos de mujer antes de los 13 años de edad. Schott (1995), encontró que los artículos femeninos de mayor preferencia en estas primeras experiencias lo eran la ropa interior, las medias "nylon" y los peinados femeninos.

Un hallazgo significativo en el estudio realizado por Schott (1995), está relacionado con la posición que ocupaban los transvestis entrevistados en la familia de origen. Este concluyó que el orden de nacimiento de esta muestra, en su mayoría el hijo mayor o único, influyó en la conducta de transvestismo en estos hombres. Por lo tanto, según Schott (1995), los niños que ocupan la posición de hijo mayor o son hijos únicos, están en riesgo de desarrollar comportamiento transvestista. Cabe señalar que esto está relacionado en cómo las madres intentaban compensar sus problemas maritales con su hijo mayor. Desde la perspectiva de la teoría sistémica, la ansiedad producida por un matrimonio insatisfecho puede ser amortiguada al adentrar al hijo en una relación de madre/padre/hijo (Schott, 1995).

Varios estudios reflejan que los transvestis se visualizan a sí mismos como heterosexuales (Pomeroy, 1999; Bullough y Bullough, 1997). Bullough y Bullough (1997), encontraron que la edad promedio de los 372 transvestis entrevistados era de 48 años, al momento del estudio. De esta muestra, el $64 \%$ de ellos estaba casado o vivía con alguna mujer, el $34 \%$ vivía solo y el $2 \%$ vivía con un hombre.

Otra variable estudiada es las relaciones paterno y materno filiales de los transvestis. Schott (1995), encontró que en las relaciones parentales de estos individuos, la influencia maternal a la que fueron expuestos era mayor que la paternal. Los transvestis reportaron tener una relación más cercana y positiva con su madre que con su padre. La mayor parte de la muestra expresó que sus padres eran poco afectuosos con ellos, despreocupados por sus tareas, emocionalmente ausentes de sus vidas, e inclusive, abusivos. Informaron que las relaciones con sus padres fluctuaban de neutrales a negativas. Estos hombres percibían a sus madres como accesibles y de mucho apoyo emocional para ellos, describiendo las relaciones maternales desde neutrales a positivas (Schott, 1995). Además, dicho investigador halló que problemas en la relación marital de la madre eran compensados a través de la relación con su primer hijo, viéndolo como un vehículo de expresión de sus necesidades de afecto e intimidad insatisfecha.

Estudios efectuados acerca del transvestismo también han auscultado sobre vestimentas, prácticas sexuales llevadas a cabo y la 
clientela de los transvestis (Talamini, 1982; Peo, 1988; Schott, 1995; Bullough y Bullough, 1997). En términos generales, los transvestis se caracterizan por su maquillaje excesivo, exageración en los gestos femeninos, utilización de ropa extremadamente llamativa, cabello excesivamente arreglado y llamativo y la utilización de zapatos extravagantes y muy altos. Por lo general, los transvestis tratan de exaltar lo que por regla general se consideran características femeninas, tanto en apariencia como en comportamiento.

Boles \& Elifson (1994), realizaron un estudio acerca de la organización social y las prácticas llevadas a cabo por transvestis prostitutos en tres áreas geográficas de Atlanta. El estudio reflejó que en dos de las áreas, la mayor parte de la clientela eran hombres que desconocían la identidad sexual de los transvestis y por el contrario, buscaban prostitutas femeninas y se confundían. Por esta razón, los transvestis practicaban más el sexo oral, para así no ser descubierta su propia identidad sexual. Los ingresos económicos que generaban los transvestis fluctuaban entre $\$ 265$ a $\$ 450$ semanales. La mayoría de ellos llevaba aproximadamente diez años en la prostitución (Boles \& Elifson, 1994).

El uso de protección durante el acto sexual era variado. Los prostitutos que generaban menos ingresos, generalmente no utilizaban protección alguna, mientras que los que generaban mayor ingreso sí utilizaban condones. Los investigadores encontraron que el conocimiento que poseían acerca del SIDA también era variado. Los que generaban más dinero, usualmente tenían mayor información acerca del SIDA que los demás (Boles \& Elifson, 1994). Por último, estos autores concluyeron que la condición del HIV está conectada al compromiso con el transvestismo, ya que este alto compromiso los lleva al aislamiento social y/o físico, participación de conductas sexuales arriesgadas (por ejemplo: relaciones anales receptivas sin protección) y experiencias de vida adversas con sentimientos de vulnerabilidad y desesperanza (Boles \& Elifson, 1994).

\section{Metodología}

En el presente estudio se utilizó un diseño exploratorio descriptivo. El universo del estudio estaba constituido por la totalidad de los transvestistas masculinos de "tabla" y de "calle" del área metropolitana de San Juan, Puerto Rico. Se seleccionó una muestra no probabilística de 27 transvestistas masculinos por 
disponibilidad. De éstos 15 eran transvestis de "calle" y 12 fueron transvestis de "tabla". El tamaño de la muestra se justifica ya que se desconoce la cantidad exacta de transvestistas masculinos que existen en el área metropolitana de San Juan, Puerto Rico.

Para la recopilación de datos se diseñó una planilla elaborada por la autora y sus estudiantes, la cual fue suministrada a través de entrevistas personales. Se identificaron las áreas donde se reúnen y frecuentan los transvestis y se realizaron visitas a las mismas con el fin de realizar las entrevistas. Dichas áreas identificadas fueron: la Parada 15 de Santurce y varias discotecas en el área de Santurce y San Juan. Los entrevistadores fueron esta autora y sus estudiantes del curso TSOC 6279 - Sexualidad Humana y Trabajo Social, del Segundo Semestre del Año 1997-98. Las entrevistas se completaron en mayo de 1998.

\section{Hallazgos del estudio}

Las preguntas dirigidas a los transvestis masculinos entrevistados iban dirigidas a explorar las siguientes áreas: características socio-demográficas; autopercepción y primeras experiencias de transvestismo, relaciones familiares y parentales; características de su clientela o público; prácticas sexuales utilizadas y relaciones de pareja.

\section{Características sociodemográficas}

Con relación a la edad de los entrevistados, los resultados reflejaron que existe una diferencia significativa entre los transvestis de calle y los de tabla. El $87 \%$ de los transvestis de calle se encontraban entre las edades de 16 a 25 años, mientras que la totalidad de los transvestis de tabla reportaron tener más de 31 años de edad, concentrándose la mayoría (67\%) entre 36 a 40 años.

El 53\% de los transvestis de calle informaron tener aprobado la escuela superior, mientras que un $40 \%$ reportó poseer un grado asociado o algunos créditos universitarios aprobados. Sin embargo, la gran mayoría (75\%) de los transvestis de tabla habían realizado algún estudio universitario.

Todos los entrevistados, tanto los de calle como los de tabla señalaron ser solteros al momento del estudio. El 47\% de los transvestis de calle reportó vivir con una amiga y el $20 \%$ vivir sólo. La misma tendencia se observó en los transvestis de tabla: $42 \%$ vivía con una amiga y $42 \%$ informó vivir solo. La minoría reportó vivir con su 
madre o ambos padres.

La mayoría de los transvestis de calle $(60 \%)$ informó pertenecer a la religión católica, mientras que el $100 \%$ de los transvestis de tabla se ubicó en la religión católica.

El 40\% de los transvestis de calle reportó no realizar trabajo asalariado durante el día, contrario a el $83 \%$ de los transvestis de tabla manifestó dedicarse a un trabajo asalariado, entre los que se encuentran estilismo, diseño y costura.

\section{Autopercepción y primeras experiencias de transvestismo}

Se le solicitó a los entrevistados que le dieran significados a los siguientes conceptos: homosexual, transexual y transvestista. Los resultados reflejan que la mayoría de los transvestis de calle no tienen claro la diferencia entre los conceptos transexual y transvesti. Algunos de los significados que ofrecieron para transexual fueron, entre otros, los siguientes: "hombre que se vista de mujer"; "persona que deja de ser quién es para caracterizar el sexo opuesto"; "viste de mujer y tiene senos"; "el que de día es hombre y de noche es mujer", etc. Algunos relacionaron el término con la utilización de hormonas. Por otro lado, la mayoría expuso que para ellos el transvestista guardaba relación con la persona que se viste de mujer para ofrecer un "show" o entretener al público. Cabe destacar que diversos autores definen el transexualismo como la acción compulsiva y obsesiva de convertirse en un miembro del sexo opuesto por intervenciones quirúrgicas (McCary \& McCary, 1997; Gotwald \& Golden, 1983).

La confusión en los conceptos transexual y transvesti se reflejó en la pregunta de cómo estos sujetos se consideraban o percibían. La mayoría (53\%) de los transvestis de calle se ubicó en la categoría de transexual (realmente ellos no se habían practicado operaciones quirúrgicas), mientras que un gran número $(40 \%)$ se percibió como homosexual. Ninguno de los transvestis de tabla se consideraba heterosexual.

El grupo de transvesti de tabla tenía claro la diferencia entre los conceptos transexual y transvestista. La totalidad de los transvestis de tabla se percibió como homosexual.

Los transvestis de calle expresaron que los aspectos que le motivaban a ser transvesti eran:

- "admirar la belleza física de la mujer y la habilidad que ésta tiene para entender las cosas” 
- "por necesidad o dinero"

- "se nace así, desde chiquito se comienza"

- "problemas en el hogar"

- "amistades que influyen"

Por otro lado, en su mayoría los transvesti de tabla manifestaron que la motivación que los lleva a vestirse con ropas del sexo opuesto estriba en el arte que esto envuelve, además de la actuación y aplausos que éstos reciben.

Los transvestis de calle, en su mayoría, expresaron que la experiencia que más influyó en ellos para tener esta conducta estuvo relacionada con su niñez, ya que en dicha etapa: "les llamaba la atención las cosas que caracterizaban a una mujer, se percibían diferentes desde pequeños y le gustaban los niños. Otros expresaron que la experiencia con sus "amistades con las que se pasaban" influyó en ellos. Por otro lado, los transvestis de tabla manifestaron que lo que más les influyó fue su deseo de trabajar en el escenario como artistas, aunque también expresaron influencia de amistades.

En la Tabla \#1 se desglosa el período en que los transvestis sintieron el deseo de vestirse con ropa del género opuesto. Se destaca que los transvestis de calle sintieron el deseo durante la infancia y adolescencia, contrario a los de tabla, lo cual ocurrió en la adultez. Esta misma tendencia en ambos grupos se refleja en la edad en que se vistieron de mujer (Tabla \#2).

Tabla \# 1

Período en que comenzó el deseo de vestirse con ropas del género opuesto

\begin{tabular}{lrrrr}
\hline \multirow{2}{*}{$\begin{array}{l}\text { Período que comenzó } \\
\text { el deseo }\end{array}$} & \multicolumn{4}{c}{ Transvestis } \\
\cline { 2 - 6 } & \multicolumn{2}{c}{ Calle } & \multicolumn{2}{c}{ Tabla } \\
\cline { 2 - 6 } & F & $\%$ & F & $\%$ \\
\hline Infancia & 5 & 33 & 1 & 8 \\
Adolescencia & 9 & 60 & 3 & 25 \\
Adultez & 1 & 7 & 8 & 67 \\
\hline \multicolumn{1}{c}{ Totales } & 15 & 100 & 12 & 100 \\
\hline
\end{tabular}




\section{Tabla \#2}

Edad en que se vistieron de mujer por primera vez

\begin{tabular}{lrrrr}
\hline & \multicolumn{5}{c}{ Transvestis } \\
\cline { 2 - 5 } Edad & \multicolumn{3}{c}{ Calle } & \multicolumn{2}{c}{ Tabla } \\
\cline { 2 - 5 } & F & $\%$ & F & $\%$ \\
\hline $5-9$ & 4 & 26 & 0 & 0 \\
$10-14$ & 1 & 7 & 0 & 0 \\
$15-19$ & 6 & 40 & 2 & 17 \\
$20-24$ & 3 & 20 & 7 & 58 \\
$25-29$ & 1 & 7 & 3 & 25 \\
\hline \multicolumn{1}{c}{ Totales } & 15 & 100 & 12 & 100 \\
\hline
\end{tabular}

Los artículos más preferidos, al principio de vestirse con ropas del género opuesto, tanto para los transvestis de calle como los de tabla fueron: aquellos relacionados al maquillaje, los zapatos y los vestidos. Los transvestis de calle también preferían la ropa interior, las prendas y los accesorios para el cabello. El otro grupo no manifestó preferencia por estos útlimos artículos.

La gran mayoría de ambos grupos señalaron que percibían que su personalidad cambiaba al vestirse de mujer. Al explorar sobre el significado que esta población le daba al vestirse con ropas del otro género, ambos grupos realizaron expresiones, tales como:

- "me siento mujer"

- "me siento a gusto, completa"

- "me siento mujer y pienso como mujer"

- "me siento feliz y va acorde con mi personalidad"

- "soy algo que quisiera ser"

Por último, los sujetos del estudio habían percibido rechazo por su estilo de vida, lo cual refleja claramente una similitud entre ambos grupos (Tabla \#3) 
Tabla \#3

Percepción de rechazo por su estilo de vida

\begin{tabular}{crrrrr}
\hline \multirow{2}{*}{ Percepción de Rechazo } & \multicolumn{4}{c}{ Transvestis } \\
\cline { 2 - 5 } & \multicolumn{2}{c}{ Calle } & \multicolumn{2}{c}{ Tabla } \\
\cline { 2 - 5 } & F & $\%$ & F & $\%$ \\
\hline \multirow{2}{*}{ Sí } & 12 & 80 & 11 & 92 \\
No & 3 & 20 & 1 & 8 \\
\hline & Totales & 15 & 100 & 12 & 100 \\
\hline
\end{tabular}

Las personas de las cuales han sentido rechazo son en orden de frecuencia: compañeros de trabajo, heterosexuales, padres y hermanos.

Relaciones familiares y parentales

La Tabla \#4 refleja la posición que ocupaban los entrevistados en su familia de origen. Nótese que los transvestis de calle, en su mayoría, (47\%) eran el hijo del medio, mientras que el $58 \%$ de los de tabla ocupaban la posición del hijo mayor. Ninguno de los entrevistados resultó ser hijo único en su familia de origen.

\section{Posición en la Familia de Origen}

\begin{tabular}{lrrrrr}
\hline \multirow{2}{*}{ Posición } & \multicolumn{4}{c}{ Transvestis } \\
\cline { 2 - 4 } & Falle & Tabla & \\
\cline { 3 - 5 } & F & $\%$ & F & $\%$ \\
\hline Hijo mayor & 5 & 33 & 7 & 58 \\
Hijo del medio & 7 & 47 & 2 & 17 \\
Hijo menor & 3 & 20 & 3 & 25 \\
\hline \multicolumn{1}{c}{ Totales } & 15 & 100 & 12 & 100 \\
\hline
\end{tabular}

Los padres de los entrevistados fueron descritos por éstos como fuertes de carácter, estrictos, moralistas, agresivos, mientras que a 
la madre la visualizaban como una persona buena y tranquila, bella, flexible y que representa amor y entendimiento.

En ambos grupos de entrevistados sus padres/madres conocían sobre su estilo de vida. La totalidad de los transvestis de tabla piensan que sus padres/madres se sienten bien con su estilo de vida, sin embargo, entre los transvestis de calle, la mayoría manifestó que sus padres se mostraban preocupados y rechazaban la conducta de prostitución.

La gran mayoría de los transvestis perciben muy positiva y positiva su relación con su madre. Ninguno de éstos evaluó esta relación como negativa.

Tabla \#5

Percepción de la relación con la madre

\begin{tabular}{lcccc}
\hline \multirow{2}{*}{ Relación con la madre } & \multicolumn{4}{c}{ Transvestis } \\
\cline { 2 - 5 } & \multicolumn{2}{c}{ Calle } & \multicolumn{2}{c}{ Tabla } \\
\cline { 2 - 5 } & F & $\%$ & F & $\%$ \\
\hline Muy positiva & 8 & 53 & 5 & 47 \\
Positiva & 3 & 20 & 6 & 50 \\
Neutral & 1 & 7 & 1 & 8 \\
No aplica $^{1}$ & 3 & 20 & 00 & 00 \\
\hline \multicolumn{1}{c}{ Totales } & 15 & 100 & 12 & 100 \\
\hline
\end{tabular}

${ }^{1}$ Madre no vive

Por otro lado, un gran número de los transvestis, tanto de tabla como de calle expresaron que percibían como neutral su relación con sus padres (Tabla \#6). Sin embargo, se reflejó que algunos transvestis evaluaron la relación paternal como muy positiva y positiva. 


\section{Tabla \#6}

Percepción de la relación con el padre

\begin{tabular}{|c|c|c|c|c|}
\hline \multirow{3}{*}{ Relación con el padre } & \multicolumn{4}{|c|}{ Transvestis } \\
\hline & \multicolumn{2}{|c|}{ Calle } & \multicolumn{2}{|c|}{ Tabla } \\
\hline & $\mathbf{F}$ & $\%$ & $\mathbf{F}$ & $\%$ \\
\hline Muy positiva & 0 & 0 & 1 & 8 \\
\hline Positiva & 3 & 20 & 2 & 17 \\
\hline Neutral & 6 & 40 & 4 & 33 \\
\hline Negativa & 1 & 7 & 0 & 0 \\
\hline Muy negativa & 2 & 13 & 1 & 8 \\
\hline No aplica $^{1}$ & 3 & 20 & 4 & 33 \\
\hline Totales & 15 & 100 & 12 & 100 \\
\hline
\end{tabular}

${ }^{1}$ Padre no vive

\section{Clientela y prácticas sexuales}

La clientela de los transvestis de calle son en su mayoría personas entre las edades de 20 a 40 años; profesionales, tales como doctores, abogados, ingenieros, empresarios, así como bomberos, electricistas y policías fueron los descritos como usuarios de los servicios de éstos. La gran mayoría de sus clientes son casados y clasificados como de clase alta y media alta. Los transvestis de tabla no pudieron precisar describir su clientela, ya que éstos alegaron no tener contacto directo con su clientela.

El tiempo que los transvestis llevan en estos negocios (prostitución o "show" de entretenimiento) resultó ser menor en los de calle que en los de tabla, lo cual se refleja en la siguiente tabla:

\section{Tabla \#7}

Tiempo que llevan en estos negocios

\section{Tiempo}

Meses

1-5 años

\begin{tabular}{rlll}
\multicolumn{3}{c}{ Transvestis } \\
\hline \multicolumn{2}{c}{ Calle } & \multicolumn{2}{c}{ Tabla } \\
\hline F & $\%$ & F & $\%$ \\
3 & 20 & 0 & 00 \\
10 & 66 & 0 & 00
\end{tabular}


Tabla \#7 (Cont.)

\begin{tabular}{|c|c|c|c|c|}
\hline \multirow{3}{*}{ Tiempo } & \multicolumn{4}{|c|}{ Transvestis } \\
\hline & \multicolumn{2}{|c|}{ Calle } & \multicolumn{2}{|c|}{ Tabla } \\
\hline & $\mathbf{F}$ & $\%$ & $\mathbf{F}$ & $\%$ \\
\hline 6-10 años & 1 & 7 & 3 & 25 \\
\hline 11-15 años & 0 & 0 & 4 & 33 \\
\hline $16-20$ & 0 & 0 & 3 & 25 \\
\hline 21 o más & 0 & 0 & 2 & 17 \\
\hline No contestó & 1 & 7 & 0 & 00 \\
\hline Totales & 15 & 100 & 12 & 100 \\
\hline
\end{tabular}

El dinero que devengan mensualmente los transvestis de tabla es un promedio inferior a los de calle. Estos últimos reportaron generar en promedio de $\$ 1,000$ a $\$ 2,000$ dólares mensuales, mientras que los transvestis de tabla reflejaron ganar $\$ 500$ o menos al mes.

Las prácticas sexuales que con más frecuencia le solicitan y realizan los transvestis de calle son, en orden de frecuencia, las siguientes: relaciones orales, relaciones anales, exhibición, masturbación, relaciones entre tres y orgías.

\section{Información u orientación sobre el SIDA}

Sólo un transvesti de calle reportó no haber recibido información u orientación sobre el SIDA. Todos los demás indicaron que obtuvieron la misma de parte de Fundación SIDA (la gran mayoría de los entrevistados), familiares, amistades, prensa, libros, folletos, a través de conferencias, talleres y charlas de prevención.

La totalidad de los transvestis de tabla utilizan condones como medida para prevenir el SIDA y otras enfermedades de transmisión sexual. Igual tendencia se observó en los transvestis de tabla, ya que el $67 \%$ informó hacer uso del condón. Sin embargo, 25\% utilizan las relaciones estables como medida de prevención y el $8 \%$ la abstinencia.

\section{Relaciones de pareja}

La gran mayoría (80\%) de los transvestis de calle manifestó no haber sostenido una relación amorosa o sexual con personas del otro sexo. Se observa una tendencia de los transvestis de tabla hacia las relaciones heterosexuales (Tabla \#8). 


\section{Tabla \#8}

Relaciones amorosas o sexual con personas de otro sexo

\begin{tabular}{cccccc} 
& & \multicolumn{3}{c}{ Transvestis } \\
\cline { 3 - 6 } Relación & \multicolumn{2}{c}{ Calle } & \multicolumn{2}{c}{ Tabla } \\
\cline { 3 - 6 } & & F & $\%$ & F & $\%$ \\
\hline \multirow{2}{*}{ Sí } & 3 & 20 & 4 & 33 \\
No & 12 & 80 & 8 & 67 \\
\hline \multirow{2}{*}{ Totales } & 15 & 100 & 12 & 100 \\
\hline
\end{tabular}

De los transvestis que habían sostenido relaciones heterosexuales, el $43 \%$ afirmó que su pareja conocía sobre su deseo y acción de vestir con ropas femeninas.

\section{Discusión}

La mayoría de los transvestis de tabla son mayores en edad comparados con los transvestis de calle. Los transvestis de tabla estaban más preparados académicamente que los de calle. Contrario a el estudio de Bullough y Bullough (1997). La totalidad de los transvestis solteros y la minoría reportaron vivir con su familia de origen. La religión que predominaba entre los transvestis fue la católica.

Los transvestis entrevistados no se perciben como heterosexuales, contrario a lo encontrado por Pomeroy (1999), y Bullough y Bullough (1997). Un gran número se autopercibe como homosexual o transexual.

La admiración por la mujer, problemas en el hogar, necesidad económica y reconocimiento a través de aplausos son algunas de las razones que motivaron a estos hombres a su conducta de transvestismo. En ambos grupos la influencia de amistades fue relevante para su primera experiencia con ropas del sexo femenino. Los transvestis de calle se vistieron con ropaje de mujer a más temprana edad que los transvestis de tabla.

Los hallazgos de este estudio no concuerdan con los reportados por Schott (1995), respecto a la posición que ocupaban los transvestis en su familia de origen. Los transvestis entrevistados ocupaban otras posiciones (hijo del medio, hijo menor) y ninguno resultó ser hijo 
único en su familia de origen. Sin embargo, los resultados concuerdan con Schott (1995) respecto a la percepción de las relaciones paterno y materno filiales. La gran mayoría de los transvestis perciben muy positiva o positiva su relación con su madre, no tanto así con la relación paternal.

Comparado con el estudio de Boles \& Elifson (1994), los transvestis de calle llevan menos tiempo en el negocio de la prostitución; sin embargo, en ambos estudios se encontró que los transvestis prostitutos en promedio generan más o menos la misma cantidad de dinero por sus servicios.

\section{Implicaciones sociales y para la práctica de trabajo social}

Este estudio contribuye a entender un poco la población de transvestis, sus experiencias y necesidades. Se espera que el mismo propicie en los profesionales de trabajo social asumir un rol más activo en la búsqueda de información y conocimientos sobre este grupo, a fin de que se puedan fortalecer la prestación de servicios hacia ellos, de así requerirlos. Es imperativo que los profesionales de ayuda comiencen a tomar conciencia de que el transvestismo es parte de la diversidad humana y como tal se deben reconocer y valorar los derechos de este grupo oprimido.

\section{Agradecimiento y reconocimiento}

Deseo ofrecer un reconocimiento muy especial a los siguientes estudiantes, quienes con su valiosa aportación y colaboración hicieron posible este estudio: Yalinette Agosto, Luz Lynnette De Jesús, Wanda I. Figueroa, Waleska Guadalupe, Lesley Jiménez, Karine Lafontant, Lis M. Milland, Betzaida Ramos, Suzanne Roig, Raúl A. Torres y Jennifer Vargas. Gracias por las noches que invertimos al recoger los datos de este estudio. 


\section{Referencias}

Barker, R. (1996). Social work dictionary (3rd ed.). Washington, DC: NAASW Press.

Boles, J. \& Elifson, K. (1994). The social organization of transvestite prostitution and aids. Social Science and Medicine, 39 (1), 85-93.

Bullough, B. \& Bullough, V. (1997). Are trasvestites necessarily heterosexuals? Archieves of Sexual Behavior, 26(1), 1-12.

De los Santos, O. (1995, abril 10). De actores transvestis y el transvestimos. El Vocero, pp. 216-220.

Gotwald, W.H. y Golden, G.H. (1983). Sexualidad: La experiencia humana. México, D.F. El Manual Moderno, S.A. de C.V.

Hyde, J.S. (1986). Understanding human sexuality (3rd ed.). New York: McGraw-Hill Book Company.

McCary, J. Y S. McCary (1996). Sexualidad humana. México, D.F. Editorial El Manual Moderno, S.A. de C.V.

Nogver, J. (1971). Diccionario enciclopédico de la educación sexual. Ed. Aura, Tomo II.

Peo, R.E. (1988). Transvestism. Journal of Social Work and Human Sexuality, $7(1)$.

Pomeroy, W. (1999). http://edu./,mberz/ttt/articles/tufacts.txt.

Schott, R. (1995). The childhood and family dinamics of transvestites. Archieves of Sexual Behavior, (3), 309-327.

Talamini, J. (1982). Boys will be girls: The hidden world of the heterosexual male transvestite. U.S.: University Press of America.

Valdes, M. y otros (1992). DSM III R, Manual diangóstico y estadístico de los trastornos mentales. Barcelona: Masson. S.A. 
